\title{
Painless Aortic Dissection Presented with Acute Paraplegia
}

\author{
ORCID: \\ Mahmoud Abdelnaby: https://orcid.org/0000-0001-8016-9049 \\ Mohammed Abada: https://orcid.org/0000-0002-2043-2973 \\ Alyaa Elsayed: https://orcid.org/0000-0002-5729-5765 \\ Moustafa Dawood: https://orcid.org/0000-0001-8509-5982 \\ Yehia Saleh: https://orcid.org/0000-0003-3873-5582 \\ Abdallah Almaghraby: https://orcid.org/0000-0001-6541-0850
}

Mahmoud Abdelnaby, Mohammed Abada ${ }^{1}$, Alyaa Elsayed², Moustafa Dawood², Yehia Saleh², Abdallah Almaghraby²

Clinical and Experimental Internal Medicine Department, Cardiology and Angiology Unit, Medical Research Institute, Alexandria University, ${ }^{1}$ Department of Critical Care Medicine, Faculty of Medicine, Alexandria University, ${ }^{2}$ Department of Cardiology, Faculty of Medicine, Alexandria University, Alexandria, Egypt

\section{Abstract}

Acute aortic dissection is considered a potentially fatal condition. Neurologic manifestations such as paraplegia are quite rare. We report a case of acute paraplegia in a hypertensive smoker chronic obstructive lung disease patient. Transthoracic echocardiography revealed a dissection flap starting at the aortic annulus and extending to the descending thoracic aorta. Computed tomographic aortography confirmed the diagnosis with a dissection flap extended from the aortic annulus to the entire distal aorta. The patient was referred to another specialized cardiothoracic center, but unfortunately, he died during the surgery.

Keywords: Aortic dissection, computed tomographic aortography, echocardiography, painless paraplegia

\section{INTRODUCTION}

Acute aortic dissection is the most common cause of death among all aortic pathological conditions. In cases of aortic dissection, the layers of the media are dissected by a column of blood through an intimal tear with variable degrees of proximal and distal extension within the length of the aorta. A wide range of symptoms and signs is linked to either rupture or occlusion of a major vessel. ${ }^{[1]}$

Pain is the most common classical symptom. Rarely, aortic dissection can be painless. In rare occasions $(2 \%-5 \%$ of the patients), acute paraplegia can manifest mainly if the intercostal arteries are involved by the aortic dissection. ${ }^{[1]}$

\section{Case Report}

A 55-year-old hypertensive chronic obstructive lung disease (COPD) heavy smoker male patient, not compliant on his medical therapy, presented with acute exacerbation

Submission: 07-Jul-18 Accepted: 03-Aug-18
Access this article online
Quick Response Code:

of COPD. On clinical examination, he was distressed and tachypneic with diffuse expiratory wheezes all over his chest and his blood pressure was 140/90 $\mathrm{mmHg}$. Arterial blood gases revealed hypoxia and severe respiratory acidosis, so he was mechanically ventilated. After 1 day, he started to improve and weaned from mechanical ventilation safely. After weaning, he started to complaint of acute onset of paraplegia without any chest or abdominal or back pain. Clinical examination revealed a motor loss in both lower limbs together with a sensory loss to the level of T9 with loss of bilateral peripheral pulsations from the femoral arteries downward. Electrocardiogram showed $0.5 \mathrm{~mm}$ ST elevation in the inferoposterior leads. Laboratory investigations revealed

Address for correspondence: Dr. Mahmoud Abdelnaby, Cardiology and Angiology Unit, Clinical and Experimental Internal Medicine Department, Medical Research Institute, University of Alexandria, Alexandria, Egypt. E-mail: mahmoud.hassan.abdelnabi@outlook.com 
elevated cardiac enzymes and renal and hepatic functions. His chest X-ray showed a wide mediastinum. Urgent transthoracic

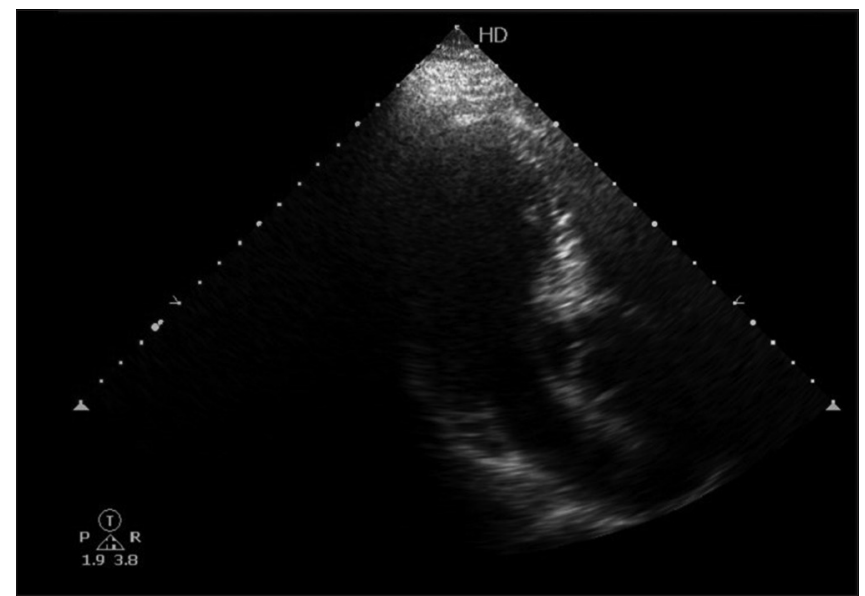

Figure 1: Transthoracic echocardiography apical long view showing a dissection flap extending through ascending aorta

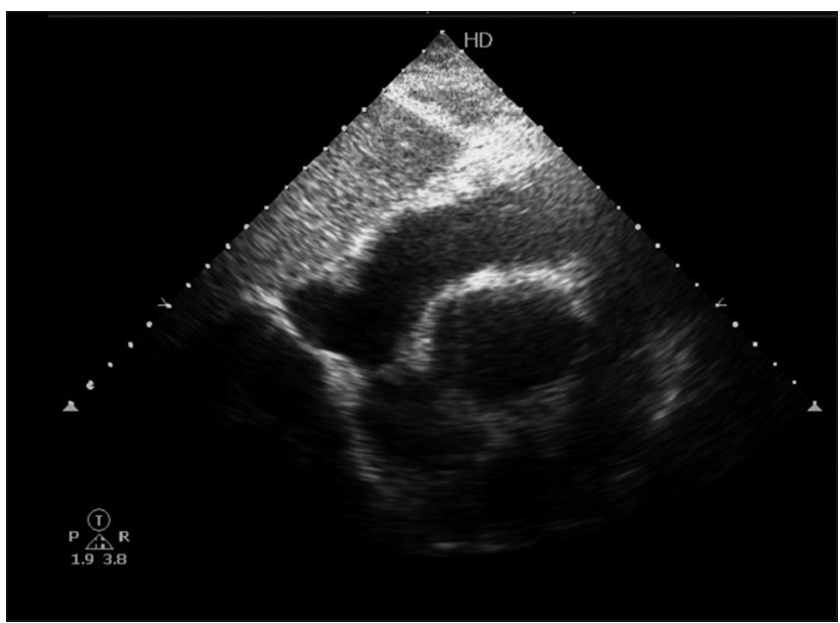

Figure 3: Transthoracic echocardiography subcostal view showing a dissection flap seen at the ascending aorta

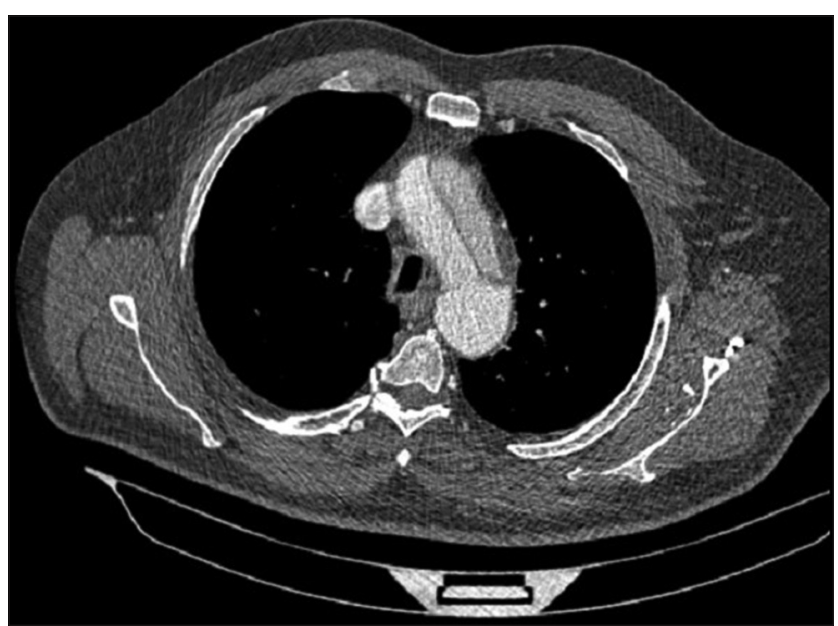

Figure 5: Computed tomographic aortography revealing a dissection flap at the aorta echocardiography [Figures 1-4] revealed a dilated aorta with a dissection flap extending from the aortic annulus to the aortic

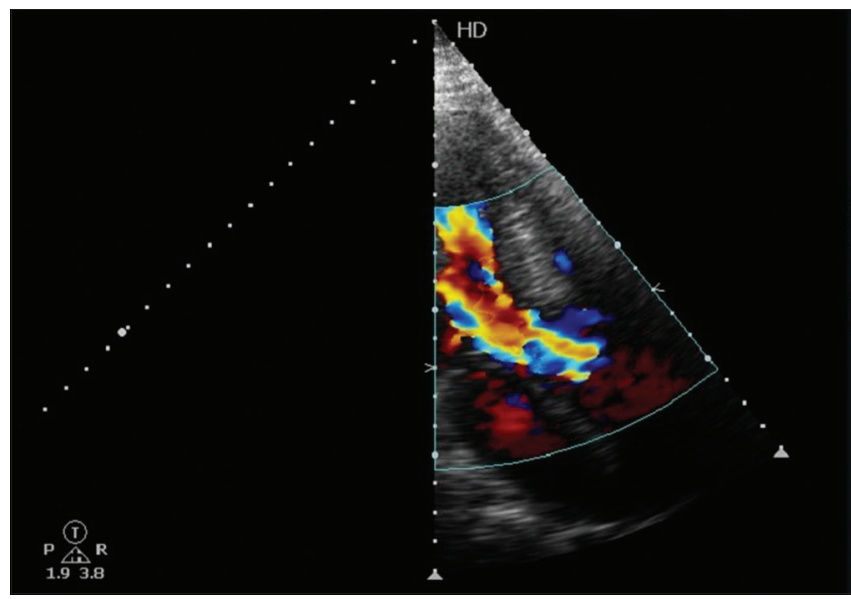

Figure 2: Transthoracic echocardiography color flow in apical long view showing severe aortic regurgitation

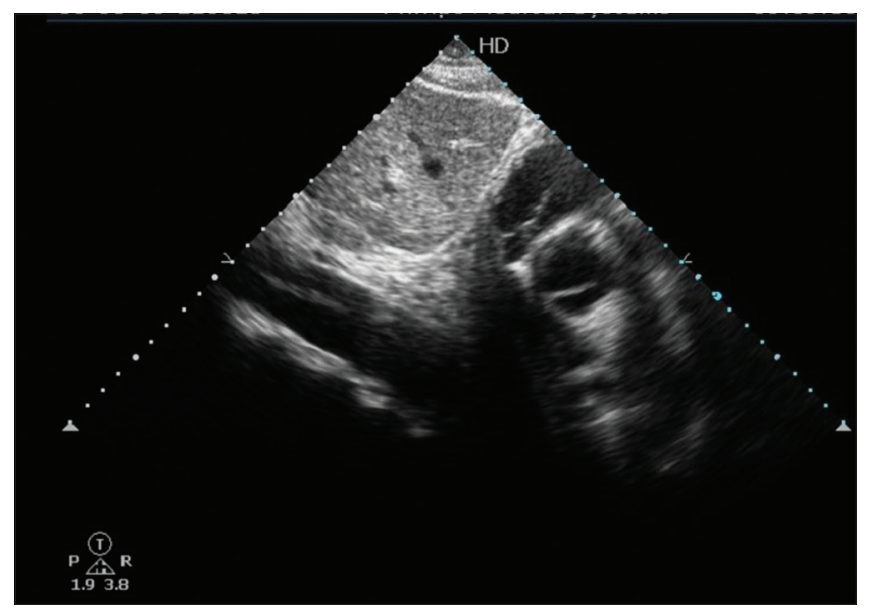

Figure 4: Transthoracic echocardiography subcostal view showing a dissection flap extending through the abdominal aorta

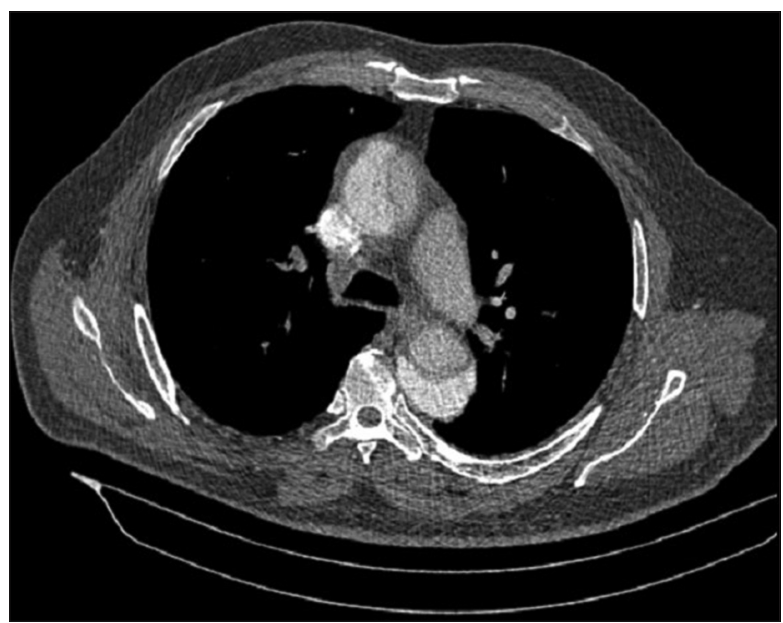

Figure 6: Computed tomographic aortography revealing a dissection flap in both ascending and descending thoracic aorta 


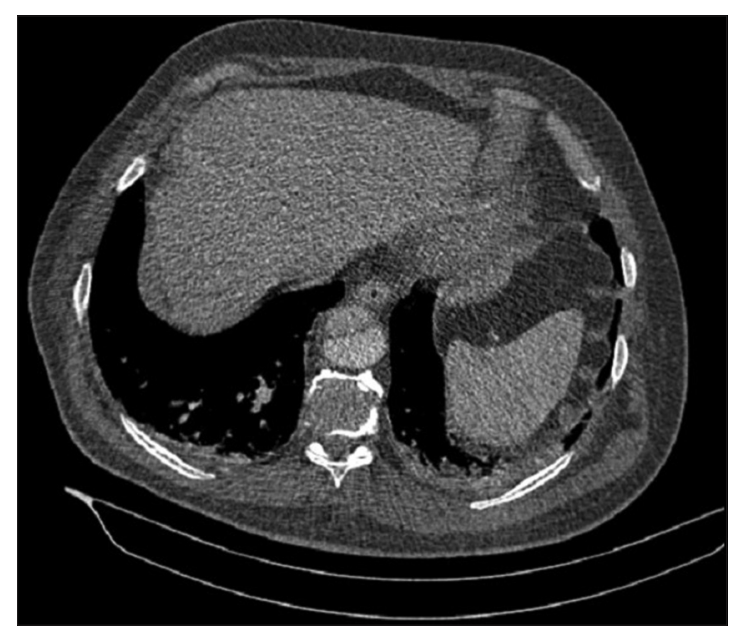

Figure 7: Computed tomographic aortography revealing a dissection flap in descending abdominal aorta

arch, the descending thoracic and the abdominal aorta with severe aortic regurgitation and a preserved left ventricular function with no regional wall motion abnormalities. Urgent computed tomographic aortography [Figures 5-7] showed an intimal tear at aortic arch extending proximally to the aortic annulus and distally to involve the entire aorta up to the left iliac artery affecting several arteries in its course, namely right coronary, innominate, left common carotid, left subclavian, intercostals, superior mesenteric, splenic, hepatic, left renal, left iliac arteries, and partially affecting right renal artery. He was referred for urgent surgery in another highly specialized cardiothoracic center where he, unfortunately, died during the operation.

\section{Discussion}

Acute aortic dissection is a sudden pathological aortic condition where blood passes through an intimal tear dissecting the layers of the aortic media, resulting in the formation of a false lumen. A wide variety of symptoms can occur according to the involved branches. A wide range of clinical presentation can be associated with aortic dissection, including confusion or coma from cerebral hypoperfusion or even stroke, electrocardiographic changes, or even elevated cardiac troponin levels, indicating cardiac hypoperfusion and myocardial dysfunction such as inferior ST-elevation myocardial infarction, loss of pulses, sensory function, or motor function due to iliofemoral or brachiocephalic hypoperfusion, creatinine elevation or low urine output due to renal hypoperfusion if the renal vessels were affected by the aortic dissection flap, paraplegia due to spinal hypoperfusion if intercostal blood vessels were affected or mesenteric hypoperfusion resulting in abdominal tenderness, bowel ischemia, or elevated liver enzymes. ${ }^{[2]}$

In $2 \%-5 \%$ of cases, paraplegia can manifest when intercostal arteries arise from the false lumen of the dissection, ${ }^{[1]}$ resulting in abrupt obstruction of the spinal arteries with interruption of blood flow to the spinal cord, especially to the crucial zones, lower thorax, and lumbar segments. ${ }^{[3]}$ Hence, ischemia of the spinal cord contributes to aortic dissection-related neurological syndromes with a sudden onset of motor weakness and paresthesia (paraplegia) ${ }^{[3]}$ and aortic dissection that presents primarily as paraplegia is rare ${ }^{[4,5]}$ Painless paraplegia is an extremely rare clinical presentation of aortic dissection with only few case reports in the literature. ${ }^{[6]}$ Aortic dissection is associated with very poor prognosis unless treated promptly. ${ }^{[7]}$ It is very crucial to differentiate between paraplegia caused by aortic dissection from other spinal vascular pathologic conditions that cause painless paraplegia. High index of suspicion, rapid diagnosis, and properly timed intervention are associated with complete recovery from aortic dissection-induced paraplegia. ${ }^{[7]}$

\section{Conclusion}

Acute paraplegia is considered a rare clinical presentation of acute aortic dissection, and it can occur even with no chest or back pain. In patients who have absent bilateral femoral pulse and abrupt onset of paraplegia even if painless, acute aortic dissection must be considered with prompt diagnosis, and urgent intervention is necessary to reduce morbidity and mortality of this fatal condition.

\section{Declaration of patient consent}

The authors certify that they have obtained all appropriate patient consent forms. In the form the patient(s) has/have given his/her/their consent for his/her/their images and other clinical information to be reported in the journal. The patients understand that their names and initials will not be published and due efforts will be made to conceal their identity, but anonymity cannot be guaranteed.

\section{Financial support and sponsorship Nil.}

\section{Conflicts of interest}

There are no conflicts of interest.

\section{References}

1. DeBakey ME, McCollum CH, Crawford ES, Morris GC Jr., Howell J, Noon GP, et al. Dissection and dissecting aneurysms of the aorta: Twenty-year follow-up of five hundred twenty-seven patients treated surgically. Surgery 1982;92:1118-34.

2. Geirsson A, Szeto WY, Pochettino A, McGarvey ML, Keane MG, Woo YJ, et al. Significance of malperfusion syndromes prior to contemporary surgical repair for acute type A dissection: Outcomes and need for additional revascularizations. Eur J Cardiothorac Surg 2007;32:255-62.

3. Gerber O, Heyer EJ, Vieux U. Painless dissections of the aorta presenting as acute neurologic syndromes. Stroke 1986;17:644-7.

4. Zull DN, Cydulka R. Acute paraplegia: A presenting manifestation of aortic dissection. Am J Med 1988;84:765-70.

5. Kwon JY, Sung JH, Kim IS, Son BC. Painless dissecting aneurysm of the aorta presenting as simultaneous cerebral and spinal cord infarctions. J Korean Neurosurg Soc 2011;50:252-5.

6. Hdiji O, Bouzidi N, Damak M, Mhiri C.Acute aortic dissection presenting as painless paraplegia: A case report. J Med Case Rep 2016;10:99.

7. Colak N, Nazli Y, Alpay MF, Akkaya IO, Cakir O. Painless aortic dissection presenting as paraplegia. Tex Heart Inst J 2012;39:273-6. 